\title{
地球自转速率变化与中国大陆 地震韵律的关系
}

\author{
赵铭 \\ (中国科学院上海天文台,上海 200030)
}

\section{关镜词地球自转、地震韵律、中国大陆}

近二十多年来, 地球自转速率变化和地震活动之间的关系已有许多文章讨论过. 许多人 都注意到地球自转加速期和减速期中的地震活动特征. 有证据表明, 有的地震带在加速期易 发生地霞, 而有的则相反, 在减速期易发地震. 我国许多学者对中国大陆及邻近地区的地震活 动的研究发现, 这个地区的地震活动存在近 20 年的周期, 10 年左右为活跃期, 另 10 年左右为 平静期, 这种韵律称为地震幕, 它在其他地震带上也有体现. 由于太阳活动的极性周期平均为 22 年, 地球自转速率变化的谱上也给出近 20 年的周期, 人们很自然地把它们联系在一起, 认 为太阳活动和地球自转的准 20 年变化可能对地震幕的形成起作用 ${ }^{[1-33,1), 2)}$ 这一想法的事实依 据仅是这几个资料序列的功率谱上均出现了近 20 年的谱峰.

但是, 谱分析方法给出的周期是整个资料段中的平均周期, 相应的振幅也是平均振幅. 如 果两个序列中有一个周期相近的谱峰, 但两者的该频率分量的时变特性却相差甚远, 我们仍然 不能设想两者会有因果联系. 因此, 本文着重于分析各频率分量的时变特性及其与地震韵律 的关系.

\section{一、日长增旦 (LOD) 序列与太阳黑子相对数 (SP) 序列的滤波}

地球自转速率变化可以用每个平太阳日中所含原子时秒数减去 86400 后的余数来表征, 这个余数称为日长增量, 常用 LOD 表示. LOD 的数值越大, 表示自转越慢. LOD 的数值 可由

$$
\mathrm{LOD}=\frac{d}{d \mathrm{AT}}(\mathrm{UT}-\mathrm{AT})
$$

得到. 世界时 UT 和原子时 AT 之差由天文观测给出. 在原子钟使用之前, 作为标准钟的时 间尺度是历书时 ET. 历书时是由太阳系天体的地心视运动体现的时间尺度, 可以通过月亮 的观测而得到. LOD 的变化含有各种尺度的分量, 短则几天, 长则几十年、几百年以至更长 的时间尺度. 本文所要研究的是几十年尺度的变化(所用的 LOD 序列取自 BIH Report for $1987)$, 从 1800 年到 1987 年,每年一值.

人们在分析一个时间序列时, 常常用谱分析方法给出时间序列在频率域中的平均特征.

本文 1989 年 12 月 27 日收到.

1) 沈宗丕,我国西部地区未来强震高潮的预测,全国第二届天地生相互关系讨论会, 1986.

2) 高建国, 圣安德烈斯地震带强震迁移和太阳活动22年等天文因素的关系, 全国第二届天地生相互关系讨论会, 1986. 
在几十年尺度上, LOD 序列在 $10 、 20 、 30 、 45 、 60$ 及 100 年附近均出现谱峰. 然而灌分沂的 结果并不能给出各分量的时变特性. 为解决这个问题, 我们用带通滤波器 ${ }^{[4]}$ 处理了 LOD 序 列。结果表明,现有资料不能证明 LOD 中存在近 100 年的周期变化,图 $1 \mathrm{a}$ 表明,百年尺度的 变化仅是一种不规则起伏. 同时,图 $1 \mathrm{~b}$ 表明, LOD 的 11 年分量不是一种周期变化, 仅是不 规则的波动. 图 2 表明 LOD 中含有较稳定的准 60 、准 40 和准 30 年周期(图 $2 a 、 b 、 c$ ), 它们 的平均周期分别为 $62 、 43$ 和 32 年. 也存在一个准 20 年周期, 但其振幅似乎受到调制, 其平均 周期为 22 年 (图 2d).

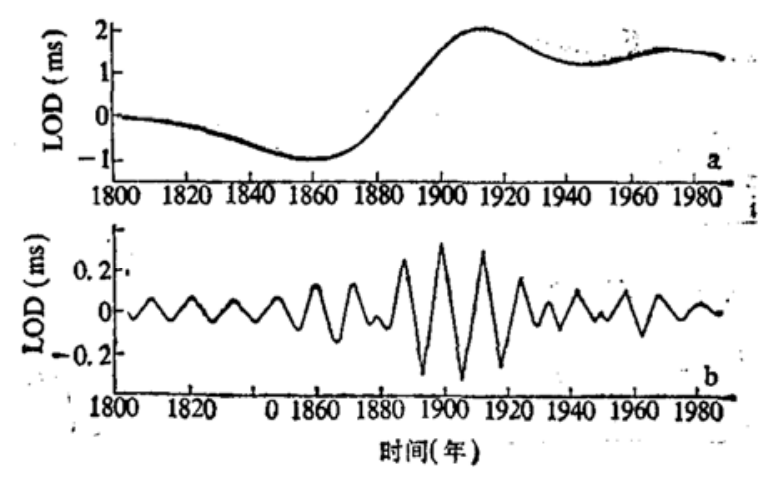

图 1

a. LOD 的百年波动; b. LOD 的准 10 年波动
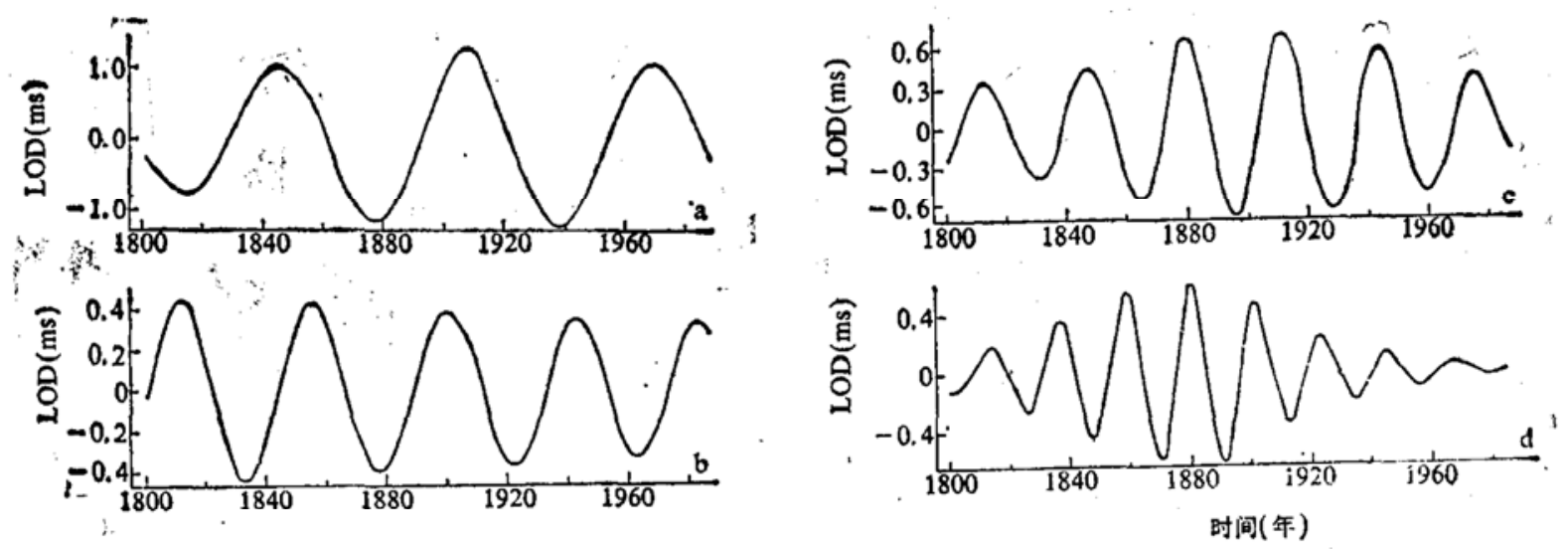

图 2

a. LOD 的准 60 年项; b. LOD 的准 40 年项; c. LOD 的准 30 年项; d. LOD 的准 20 年项

SP 序列取自文献 [5],其主要成分是近 11 年的太阳周, 其余的分量要小得多, 所以 SP 的 11 年分量和 SP 序列本身很相近.

\section{二、中国大陆地霆韵律与 LOD 及 SP 序列的关系}

从上个世纪末开始有较完整的地震记录以来的资料表明，中国大陆及邻近地区的地震按 时间的分布是不均匀的, 它存在明显的准 20 年韵律, 称为地震幕. 10 年左右为活跃幕, 10 年 左石为平静幕, 活跃幕与平静幕交替出现. 本世纪已经历了四个活跃幕和四个平静幕, 第五个 活跃幕已从 1985 年开始. 对各幕起止时间的划分, 不同作者的结果略有不同. 取文献 [6-8] 的平均,给出前四幕的活跃期为 1897-1912、1920-1934、1946-1957、1966-1976 年. 
图 3a 显示出 1880-1987 年的 LOD 序列, 图中阴影表示地震活跃期. 可以看出, 活跃期 与 LOD 的开段 (自转减速期)或降段 (自转加速期)之间并无固定对应关系. 可以认为, 中国 大陆地霞活跃性并不明显取决于地球自转的加速或减速.

图 3b 是 LOD 的准 20 年分量. 虽然它的周期和地震幕的平均间隔相近, 但从图中看到, 两者的位相关系并不固定. 而且, 近一百年中, LOD 的准 20 年项的振幅衰减了 10 倍, 但地 霞韵律依旧. 可以认为, 准 20 年的地震幕的成因并不是 LOD 的准 20 年分量.

图 3c 是太阳黑子相对数序列, 它的平均周期近 11 年. 虽然地震幕间隔与太阳活动双周 期相近, 但从图中可见,两者位相关系并不固定. 第一活跃幕开始于单周降段中点附近, 结束 于双周后的谷值年附近. 第二幕与第一幕相似. 第三活跃幕开始于双周前的谷值年附近, 结 束于后一个单周升段中点附近. 第四幕开始于双周前的谷值年附近, 结束于双周后的谷值年 附近. 由此可见,地震幕与太阳周关系也不密切.
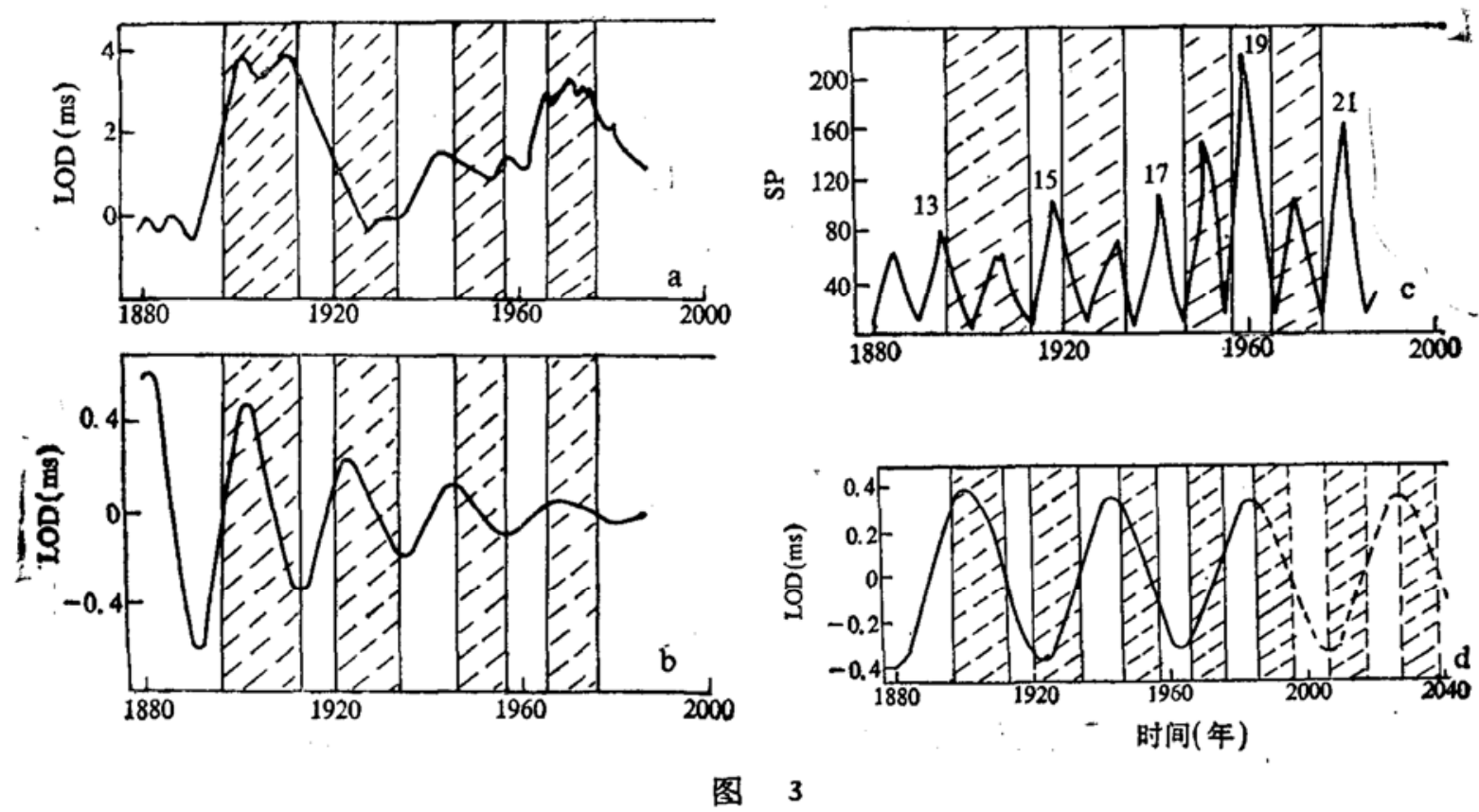

a. LOD 序列与地震幕; b. LOD 准 20 年分量与地震幕;

c. SP 序列与地震幕; d. LOD 准 40 年项与地震幕

图 3d 显示 LOD 的准 40 年分量. 可以看到, 它与地震活跃幕有很好的对应关系. 本世 纪 5 个活跃幕均开始于准 40 年分量的极值年(极大或极小)附近. 并且, 若活跃幕超前于极值 年开始, 活跃期长 (第一、二幕). 若开始于极值年之后, 则活跃期短 (第三、四幕). 第五幕开始 于极值年之后, 按上述规律预测, 将持续到 1996 年前后, 活动程度与第三、四幕相当, 弱于第 一、二幕. 图 3d 中的虚线是 LOD 准 40 年波的预测值, 据此, 我们估计下个世纪的前两个地 霞活跃幕可能分别开始于 2005 和 2025 年前后.

\section{三、关于机制问题的讨论}

地球自转的加速度在不同尺度上的数值列于表 1 , 其中对于长期项, 给出的是平均相对加 速度,对周期项,给出最大相对加速度.

由表 1 可见, 就加速度的大小来说, 准 40 年项并不是最大的. 它不仅明显小于其他几个 
表 1 不同尺度上地球自转的加速度

\begin{tabular}{c|c||c|c}
\hline 时间尺度 & $\dot{\omega} / \omega$ (平均值) & 时间 尺 度 & $\dot{\omega} / \omega$ (最大值) \\
\hline 近三千年 & $-20 \times 10^{-11} /$ 年 & 准 60 年项 & $\pm 137 \times 10^{-11} /$ 年 \\
近五亿年 & $-23 \times 10^{-11} /$ 年 & 准 40 年项 & $\pm 70 \times 10^{-11} /$ 年 \\
1800 年以来 & $-17 \times 10^{-11} /$ 年 & 准 30 年项 & $\pm 150 \times 10^{-11} /$ 年 \\
$1800-1870$ 年 & $+10 \times 10^{-11} /$ 年 & & \\
$1870-1910$ 年 & $-91 \times 10^{-11} /$ 年 & 准 20 年项 & $\pm 160 \times 10^{-11} /$ 年 \\
1910 年以来 & $+6 \times 10^{-11} /$ 年 & 综合结果 & $\pm 300 \times 10^{-11} /$ 年 \\
\hline
\end{tabular}

准周期项的最大加速度, 更远小于 LOD 本身的加速度 (约 $\pm 300 \times 10^{-11} /$ 年), 这是各周期分 量的综合结果. 然而只有准 40 年项与地震韵律有密切关系. 这个事实说明, 如果说地球自转 速度变化对中国大陆及邻区的地震活动有激发作用的话, 那么加速度的大小永不是决定性因 秦.

我们设想加速度的周期性变化对不同构造块有不同作用, 因不同构造块有不同的固有频 率. 对于自转变化周期与地块固有周期相近的情况, 将发生共振效应. 这时, 即使自转加速度 较小, 也会发生大的响应而引起地震活动. 这就是说, 中国大陆及邻区这块地方, 可能具有近 40 年的固有振动周期, 它对自转速率准 40 年变化发生共振响应而触发地震活动期.

假如以上设想符合实际, 那么地球上一定还会有另一些地块, 它们的地震活动韵率与自转 变化的另一些周期项有相关关系. 这正是我们要进一步研究的问题.

\section{四、结论}

中国大陆及邻近地区地震活跃幕的出现与地球自转速率变化的准 40 年分量密切相关, 每 次活跃幕均开始于准 40 年分量的极值年附近. 活跃幕开始得越早, 其持续时间越长, 地震活 动越激烈.

目前的第五幕预计结束于 1996 年前后, 本幕地震活动规模与第三、四幕类似, 弱于第一、 二幕.

地球上不同构造块可能对 LOD 的不同频率分量的作用最灵敏, 这可能由共振效应所 致.

\section{参考文献}

[1] 张㔀珍等, 中国地震, 1(1985)，3: 22.

[2] 杜品仁,地能大形势研究的述评与形势预测, 国家地震局地质研究所, 1988 .

[3] 徐道一等, 天文地质学概论, 地质出版社, 1983,116 .

[4] 郑大伟等, 天文学报, 27(1986), 4: 368 .

[5] 张家诚等,地学数据手册, 海洋出版社, 1986, 1280.

[6] 马宗晋等, 1966-1976 中国九大地㖮, 地震出版社, 1982 .

[7] 洪时中,西北地震学报, 6(1984), 1: 54 .

18] 万宗晋等,中国地祳, 3 (1987), 1: 23. 